hep-th/0511122

\title{
Supersymmetric compactifications of heterotic strings with fluxes and condensates
}

\author{
Pantelis Manousselis $^{1}$, Nikolaos Prezas ${ }^{2}$ and George Zoupanos ${ }^{3}$ \\ ${ }^{1}$ Department of Engineering Sciences, University of Patras, \\ GR-26110 Patras, Greece \\ pantelis@upatras.gr \\ ${ }^{2}$ Institut de Physique, Université de Neuchâtel, \\ CH-2000 Neuchâtel, Switzerland \\ nikolaos.prezas@unine.ch \\ ${ }^{3}$ Physics Department, National Technical University of Athens, \\ GR-15780 University Campus, Athens, Greece \\ zoupanos@mail. cern.ch
}

\begin{abstract}
We discuss supersymmetric compactifications of heterotic strings in the presence of $\mathrm{H}$ flux and general condensates using the formalism of $G$-structures and intrinsic torsion. We revisit the examples based on nearly-Kähler coset spaces and show that supersymmetric solutions, where the Bianchi identity is satisfied, can be obtained when both gaugino and dilatino condensates are present.
\end{abstract}




\section{Introduction}

In string theory compactifications on Calabi-Yau (CY) manifolds and the corresponding dimensional reductions [1, the resulting low-energy field theory in four dimensions typically contains a number of massless chiral fields, characteristic of the internal geometry, known as moduli. These fields, arising in four dimensions as massless modes of the higher-dimensional matter fields and from the gauge-independent variations of the metric of the compact space, correspond generically to flat directions of the four-dimensional effective potential. Therefore, the values taken by the moduli in the vacuum, which in turn specify the masses and couplings of the four-dimensional theory, are left undetermined. Hence, the theory is without predictive power.

Fortunately, the moduli problem in the form described above appears only in the simplest choice of string backgrounds, where out of the plethora of closed-string fields only the metric is assumed to be non-trivial. By considering more general backgrounds involving "fluxes" [2, 3] as well as non-perturbative effects [4, 5, the four-dimensional theory can be provided with potentials for some or all moduli. The terminology "fluxes" refers to the inclusion of nonvanishing field strengths for the ten-dimensional antisymmetric tensor fields with directions purely inside the internal manifold. Therefore, the present day problem is the choice of the appropriate background which could lead to realistic and testable four-dimensional theories.

The presence of fluxes has a dramatic impact on the geometry of the compactification space. Specifically, the energy carried by the fluxes back-reacts on the geometry of the internal space and the latter is deformed away from Ricci-flatness. Then, the CY manifolds used so often in string theory compactifications cease to be true solutions of the theory. For example, the requirement that some supersymmetry is preserved implies that the internal manifold is a non-Kähler space for heterotic strings with NS-NS fluxes [6, 7, 8, 9], while it can be a non-complex manifold for type IIA strings [10, 11, 12. For type IIB strings, instead, the deviation is mild since the overall effect due to the fluxes is a conformal rescaling of the original CY solution [13, 14]. We should mention at this point that in principle we can consider compactifications on CY manifolds with fluxes too, but these are reliable only in the large-volume limit where the flux back-reaction can be consistently ignored.

A considerable amount of literature has been devoted to the problem of including appropriately the back-reaction of the fluxes on the internal manifold and constructing examples of manifolds which are true solutions of the theory. In general, these manifolds have nonvanishing torsion. Consequently, demanding that the low-energy theory is supersymmetric implies that the internal manifold admits a $G$-structure [15]. The existence of a $G$-structure is a generalization of the condition of special holonomy. Subsequently, the allowed $G$-structures can be classified in terms of their intrinsic torsion classes [16]. 
For heterotic strings, requiring that supersymmetry is preserved in the presence of nonperturbative effects such as gaugino condensation [17, leads to $A d S_{4}$ spacetimes with noncomplex internal manifolds as potential solutions [18 ${ }^{1}$. The non-complex manifolds that we will consider here are simple homogeneous nearly-Kähler coset spaces. They were identified as interesting possible solutions of heterotic string theory in refs. [19, 20], whereas supersymmetric solutions of the form $A d S_{4} \times G / H$ were first obtained in refs. [21, 22]. In order to obtain such solutions the authors in refs. 21, 22] assumed the presence of a gaugino condensate and performed a case by case analysis. More recently, the 10-dimensional supersymmetry conditions in the presence of a non-vanishing gaugino condensate were examined in the language of $G$-structures in ref. [18.

Here, we generalize the setup of ref. [18] by considering more exotic condensates. The consideration of other condensates besides that due to the gaugino is imposed upon us for both technical and aesthetical reasons. At a technical level, the source-free Bianchi identity cannot be solved in a supersymmetry-preserving manner if only the gaugino condensate is present. One of the objectives of the present work is to show that supersymmetric solutions of the Bianchi identity can be obtained if, in addition to the gaugino condensate, a dilatino condensate acquires a non-vanishing vacuum expectation value (vev). On aesthetic grounds and although there is no known mechanism leading to condensation of the dilatino ${ }^{2}$, we adopt here a more broad point of view which considers the condensates on equal footing with the fluxes. Following this approach, we first formulate the supersymmetry conditions in the formalism of $G$-structures and then we revisit the solutions of refs. [21, 22], while completing also their list by one more example.

We should mention that, as in most works that study the supersymmetry constraints on the geometry, we do not check explicitly the equations of motion. In our case this would be a non-trivial task because the presence of the condensates renders the derivation of the equations of motion subtle [18. Instead, we rely on the fact that backgrounds that preserve some supersymmetry and where the Bianchi equations and the equations of motion for the matter fields are satisfied, are automatically solutions of the Einstein equations [12, 27]. It is straightforward to show that this is indeed true for hererotic strings by specializing the results of [27] to hererotic M-theory.

A key feature of the solutions we consider is that since they are not Ricci-flat, the fourdimensional spacetime will not be Minkowski but $A d S$ (at least in the case where some

\footnotetext{
${ }^{1}$ Originally, gaugino condensation had been suggested as a supersymmetry breaking mechanism in a Calabi-Yau compactification [4] 5]. Here, instead, we consider supersymmetric solutions on manifolds that are not Calabi-Yau.

${ }^{2}$ See however refs. 23, 24] where condensation of fermions in the gravity sector is considered in a different context and ref. [25] which discusses a similar effect in a 5-brane background. Moreover, ref. [26] considered Minkowski vacua in 11-dimensional supergravity with gravitino condensates.
} 
supersymmetry is preserved). For this reason, the Ricci-flat CY manifolds were originally more attractive candidates. Recently, however, this has become less relevant since whenever the inclusion of fluxes produces a stable vacuum without moduli, this vacuum turns out to be anti-de Sitter. Therefore, it is not a serious drawback to start from an anti-de-Sitter solution in the first place and hope that eventually non-perturbative effects will lift this to a Minkowski or de Sitter vacuum according, for example, to the scenario proposed in [28] (see also 29] for an explicit construction of this type in heterotic M-theory). This process may result in interesting GUTs in four-dimensions [30, 31, 32, 33, 34, 35.

The layout of this paper is as follows. In section 2 we establish our notation and we present the supersymmetry transformations of the fermionic fields of heterotic supergravity when various condensates are non-vanishing. In section 3 we examine the conditions imposed on the external and internal geometries under the requirement of preserving $\mathcal{N}=1$ supersymmetry in the external space. The conditions on the internal geometry are formulated in terms of the intrinsic torsion classes of the $S U(3)$-structure. In section 4 we study some specific solutions based on nearly-Kähler coset spaces. We show that the Bianchi identity can be satisfied if and only if both gaugino and dilatino condensates are present. Finally, in section 5 we present a few concluding remarks. Also, in two appendices we provide our Gamma matrix conventions and some details on the $S U(3)$-structure of the cosets under consideration.

\section{Heterotic strings with condensates}

The fields of heterotic supergravity, which is the low-energy limit of heterotic superstring theory, consists of the $\mathcal{N}=1, D=10$ supergravity multiplet which contains the fields $e_{M}^{N}, \psi_{M}, B_{M N}, \lambda, \varphi$, (i.e. namely the metric, the gravitino which is a Rarita-Schwinger field, the two-form potential, the dilatino which is a Majorana-Weyl spinor, and the dilaton which is a scalar), coupled to a $\mathcal{N}=1, D=10$ vector supermultiplet which contains the gauge field $A_{M}$ and the corresponding gaugino $\chi$. The gauge field and the gaugino transform in the adjoint of $E_{8} \times E_{8}$. The Lagrangian and the full supersymmetry variations can be found in refs. 36, 37, 38.

The supersymmetry variations of the fermionic fields, including the relevant for our discussion fermion bilinears, are $[37]^{3}$

$$
\begin{aligned}
\delta \psi_{M} & =\nabla_{M} \epsilon+\frac{\sqrt{2}}{32} \varphi^{-3 / 4}\left(\Gamma_{M} \Gamma^{N P Q}-12 \delta_{M}^{N} \Gamma^{P Q}\right) \hat{H}_{N P Q} \epsilon \\
& -\frac{1}{256}\left(\Gamma_{M} \Gamma^{N P Q}-8 \delta_{M}^{N} \Gamma^{P Q}\right)\left(\operatorname{Tr}\left(\bar{\chi} \Gamma_{N P Q} \chi\right)+\frac{1}{2}\left(\bar{\lambda} \Gamma_{N P Q} \lambda\right)\right) \epsilon+\frac{\sqrt{2}}{96}\left(\bar{\psi}_{M} \Gamma_{K L} \lambda\right) \Gamma^{K L} \epsilon,
\end{aligned}
$$

\footnotetext{
${ }^{3}$ Note that the coefficient of the last term in $\delta \lambda$ was corrected in ref. [4].
} 


$$
\begin{aligned}
\delta \chi & =-\frac{1}{4} \varphi^{-3 / 8} \Gamma^{M N} \hat{F}_{M N} \epsilon+\frac{\sqrt{2}}{64}\left(3(\bar{\lambda} \chi)-\frac{3}{2}\left(\bar{\lambda} \Gamma_{M N} \chi\right) \Gamma^{M N}-\frac{1}{24}\left(\bar{\lambda} \Gamma_{M N K L} \chi\right) \Gamma^{M N K L}\right) \epsilon, \\
\delta \lambda & =-\frac{3 \sqrt{2}}{8} \varphi^{-1} \Gamma^{M} \partial_{M} \varphi \epsilon+\frac{1}{8} \varphi^{-3 / 4} \Gamma^{M N P} \hat{H}_{M N P} \epsilon+\frac{\sqrt{2}}{384} \operatorname{Tr}\left(\bar{\chi} \Gamma_{M N P} \chi\right) \Gamma^{M N P} \epsilon .
\end{aligned}
$$

We have set the gravitational and Yang-Mills coupling constants equal to 1. This in turn implies that we work with units where $\alpha^{\prime}=4$. The supersymmetry parameter $\epsilon$ is a 10 dimensional Majorana-Weyl spinor with 16 real components. The hats denote the supercovariant generalization of the corresponding fields,

$$
\begin{aligned}
\hat{F}_{M N} & =F_{M N}-\varphi^{3 / 8}\left(\bar{\psi}_{[M} \Gamma_{N]} \chi\right), \\
\hat{H}_{M N K} & =H_{M N K}-\frac{1}{4} \varphi^{3 / 4}\left(\sqrt{2}\left(\bar{\psi}_{[M} \Gamma_{N} \psi_{K]}\right)-\left(\bar{\psi}_{[M} \Gamma_{N K]} \lambda\right)\right) .
\end{aligned}
$$

It is well-known that at the supergravity level the gauge-invariant 3-form field strength is $H_{M N P}=3 \partial_{[M} B_{N P]}-\frac{1}{\sqrt{2}} A_{[M} F_{N P]}$. The corresponding Bianchi identity reads as

$$
d H=-\frac{\sqrt{2}}{6} \operatorname{tr}(F \wedge F) .
$$

The full Bianchi identity includes one more term that is a string-theoretic correction and will be added later.

In the above supersymmetry transformations we have assumed that some of the possible condensates between the fermionic fields of heterotic string theory have non-vanishing vacuum expectation values. Our motivation for keeping only those appearing above is that, as we will see later, they permit supersymmetric solutions without rendering the analysis computationally challenging. We will also assume that the condensates $\left(\bar{\psi}_{[M} \Gamma_{N]} \chi\right)$ and $\left(\bar{\psi}_{[M} \Gamma_{N} \psi_{K]}\right)$, which appear in the supercovariant field strengths, are vanishing. We postpone the presentation of a more complete analysis incorporating all possible condensates for future work [39].

An issue that should be addressed at this point concerns the supersymmetry variations of the bosonic fields in the presence of fermion condensates. These read [37]

$$
\begin{aligned}
\delta e_{M}^{L} & =\frac{1}{2} \bar{\epsilon} \Gamma^{L} \psi_{M}, \quad \delta \varphi=-\frac{1}{3} \sqrt{2} \epsilon \lambda \varphi, \quad \delta A_{M}=\frac{1}{2} \varphi^{3 / 8} \bar{\epsilon} \Gamma_{M} \chi \\
\delta B_{M N} & =\frac{1}{4} \sqrt{2} \varphi^{3 / 4}\left(\bar{\epsilon} \Gamma_{M} \psi_{N}-\bar{\epsilon} \Gamma_{N} \psi_{M}-\frac{1}{2} \sqrt{2} \epsilon \Gamma_{M N} \lambda\right)+\frac{1}{2} \sqrt{2} \varphi^{3 / 8} \epsilon \Gamma_{[M} \operatorname{Tr}\left(\chi A_{N]}\right)
\end{aligned}
$$

and it is obvious that had the fermions acquired non-zero vevs, these variations would not vanish in general. In such a case, of course, checking the supersymmetry variations would be redundant since the non-vanishing vevs for the fermions would imply anyway broken spacetime (e.g. Lorentz) symmetry. The crucial point here is that in quantum field theory the 
fermion condensates can be non-zero while maintaining a vanishing fermion vev. Therefore, although the fermion condensates are assumed to be non-vanishing, the vevs of the corresponding fermions are taken to be zero, as is necessary for preserving maximal symmetry in spacetime. It should be emphasized that this effect has been explicitly demonstrated in $\mathcal{N}=1$ Super-Yang-Mills theory where gaugino condensation indeed takes place nonperturbatively without leading to non-zero vevs for the gauginos [40, 41]. Here, we assume that this is precisely what happens in the strongly-coupled microscopic theory that underlies our effective supergravity description. This is in line with the original treatment of gaugino condensation in hererotic supergravity where the fermion vevs are zero while a non-trivial condensate is generated [4, 5. The punchline is that the supersymmetry variations of the bosons are trivially vanishing since the fermion vevs are consistently zero.

In our setup, the only extra assumption is that some unknown quantum effects can lead to non-trivial condensates for the gravitinos. Actually, such a point of view was already taken in [26] while [25] provided some evidence that this effect indeed takes place in NS5brane backgrounds. Furthermore, we should stress that here we simply work in an effective approach where we ask what would happen if such condensates were generated. Hence, our treatment is entirely analogous to the usual chiral Lagrangian approach to hadron physics. In this approach, one simply assumes that in the IR a non-vanishing quark condensate breaking chiral symmetry is formed, although the miscoscopic theory (i.e. QCD) governing this effect is out of reach in this regime. Moreover, in order to maintain Lorentz invariance the quark vevs are assumed to be zero despite the presence of the non-perturbative quark condensate.

We now make the following field redefinitions:

$$
\begin{aligned}
\varphi & =e^{-8 / 3 \phi}, & g_{M N} & =e^{-2 \phi} g_{M N}^{(0)}, \\
\lambda & =\frac{1}{\sqrt{2}} e^{\phi / 2} \lambda^{(0)}, & \psi_{M} & =e^{-\phi / 2}\left(\psi_{M}^{(0)}-\frac{\sqrt{2}}{4} \Gamma_{M}^{(0)} \lambda^{(0)}\right), \\
\epsilon & =e^{-\phi / 2} \epsilon^{(0)}, & H_{M N P} & =\frac{3}{\sqrt{2}} H_{M N P}^{(0)}, \\
\chi & =e^{\phi / 2} \chi^{(0)}, & F_{M N} & =F_{M N}^{(0)}, \\
\Gamma_{M} & =e^{-\phi} \Gamma_{M}^{(0)}, & &
\end{aligned}
$$

with the quantities bearing the superscript (0) referring to those in (2.1). The supersymmetry variations (2.1) become

$$
\begin{aligned}
\delta \psi_{M} & =\nabla_{M} \epsilon-\frac{1}{4}\left(\hat{H}_{M}-2 \Sigma_{M}-\frac{4}{3} \Delta_{M}\right) \epsilon-\frac{1}{4} \Gamma_{M}\left(\frac{1}{3} \Sigma+\frac{1}{4} \Delta\right) \epsilon \\
\delta \chi & =-\frac{1}{4} \Gamma^{M N} \hat{F}_{M N} \epsilon+\frac{1}{32}\left(3 \Phi-\frac{3}{2} \Phi_{M N} \Gamma^{M N}-\frac{1}{24} \Phi_{M N K L} \Gamma^{M N K L}\right) \epsilon, \\
\delta \lambda & =\not \phi \epsilon+\frac{1}{24}(\hat{H}+\Sigma) \epsilon
\end{aligned}
$$


where $\Phi_{[\ldots]}=\left(\bar{\lambda} \Gamma_{[\ldots]} \chi\right)$. Notice that since these condensates are not singlets of the $E_{8} \times E_{8}$ gauge group, turning on vevs for them can break part of the original gauge symmetry. Of course, this is similar to the (partial) breaking of the original gauge symmetry due to a non-vanishing background gauge field strength.

In the field-redefinitions above we set $\left(\bar{\lambda} \Gamma_{M} \Gamma_{K L} \lambda\right)=\left(\bar{\lambda} \Gamma_{M K L} \lambda\right)$ because the difference involves terms of the form $\left(\bar{\lambda} \Gamma_{M} \lambda\right)$ which are zero due to the assumption of maximal symmetry in the external space and due to the absence of globally-defined vector fields on six-dimensional manifolds with $S U(3)$-structure. In the new variables the Bianchi identity (2.4) reads as

$$
d H=-\frac{1}{2} \operatorname{tr}(F \wedge F),
$$

with the supercovariant field strengths (2.3) being

$$
\begin{aligned}
\hat{F}_{M N} & =F_{M N}, \\
\hat{H}_{M N K} & =H_{M N K}-6 \Delta_{M N P},
\end{aligned}
$$

since after the field redefinitions we set $\left(\bar{\psi}_{[M} \Gamma_{N K]} \lambda\right)=0$. We use the standard shorthand notation $H_{M}=H_{M N P} \Gamma^{N P}, H=H_{M N P} \Gamma^{M N P}, \Sigma_{M}=\Sigma_{M N P} \Gamma^{N P}, \Sigma=\Sigma_{M N P} \Gamma^{M N P}$ with $\Sigma_{M N P}=\frac{1}{16} \operatorname{tr}\left(\bar{\chi} \Gamma_{M N P} \chi\right)$ and $\Delta_{M N P}=\frac{1}{16}\left(\bar{\lambda} \Gamma_{M N P} \lambda\right)$.

We emphasize that the metric in the above equations is the sigma-model metric and is related to the Einstein metric $g_{M N}^{0}$ as $g_{M N}=e^{-2 \phi} g_{M N}^{0}$. We have also used the fact that the covariant derivatives of a spinor with respect to these two metrics are related as $\nabla_{M} \epsilon=\nabla_{M}^{(0)} \epsilon-\frac{1}{2} \Gamma_{M}^{N} \partial_{N} \phi \epsilon$.

\section{Conditions for 4-dimensional $\mathcal{N}=1$ vacua}

\subsection{Metric ansatz and $S U(3)$-structure}

We assume that $g_{M N}^{0}$ describes the warped product $M_{1,3} \times_{w} K_{6}$ of a 4-dimensional maximally symmetric spacetime $M_{1,3}$ with a compact 6 -dimensional internal space $K_{6}$. Explicitly

$$
d s^{2}=g_{M N}^{0}(x, y) d x^{M} d x^{N}=e^{2 D(y)}\left(\hat{g}_{\mu \nu}(x) d x^{\mu} d x^{\nu}+\hat{g}_{m n}(y) d y^{m} d y^{n}\right) .
$$

Since we are interested in 4-dimensional vacua with $\mathcal{N}=1$ supersymmetry, we demand that on $K_{6}$ there exists a globally-defined complex spinor $\eta_{+}$(and its conjugate $\eta_{-}$with opposite chirality). In other words $K_{6}$ should be equipped with an $S U(3)$-structure. Our ansatz for the 10-dimensional Majorana-Weyl spinor $\epsilon$ is

$$
\epsilon(x, y)=f(y) \theta_{+}(x) \otimes \eta_{+}(y)-f^{*}(y) \theta_{-}(x) \otimes \eta_{-}(y),
$$


where $f(y)$ is an arbitrary complex function. This ansatz yields $\mathcal{N}=1$ supersymmetry in $M_{1,3}$ expressed in terms of the Weyl spinors $\theta_{ \pm}$. The 6 -dimensional spinors are normalized ${ }^{4}$ as $\eta_{+}^{\dagger} \eta_{+}=\eta_{-}^{\dagger} \eta_{-}=1$. As usual the four-dimensional spinors are taken to be Grassmann while the six-dimensional ones are commuting.

The $S U(3)$-structure is characterized by an almost complex structure and ${ }^{5}$ the associated 2 -form $J_{m n}$, and by a $(3,0)$-form $\Omega_{m n p}$. These forms are globally-defined and non-vanishing and they are subject to the following compatibility conditions:

$$
J \wedge \Omega=0, \quad \Omega \wedge \Omega^{*}=\frac{4 i}{3} J \wedge J \wedge J .
$$

Furthermore, the spinors $\eta_{ \pm}$determine (up to a phase which we fix to a convenient value) the $S U(3)$-structure forms as

$$
\begin{aligned}
J_{m n} & =-i \eta_{+}^{\dagger} \gamma_{m n} \eta_{+}=i \eta_{-}^{\dagger} \gamma_{m n} \eta_{-}, \\
\Omega_{m n p} & =\eta_{-}^{\dagger} \gamma_{m n p} \eta_{+}, \\
\Omega_{m n p}^{*} & =-\eta_{+}^{\dagger} \gamma_{m n p} \eta_{-} .
\end{aligned}
$$

Using Fierz identities one can show that indeed $J_{m}{ }^{n}$ is an almost complex structure, i.e. it satisfies $J_{m}{ }^{n} J_{n}{ }^{p}=-\delta_{m}^{p}$. Then, the projectors $\left(\Pi^{ \pm}\right)_{m}{ }^{n}=\frac{1}{2}\left(\delta_{m}{ }^{n} \mp i J_{m}{ }^{n}\right)$ can be used to separate the holomorphic and antiholomorphic parts of a generic form.

Now we can decompose the condensates in 4- and 6-dimensional pieces. First, notice the gaugino field $\chi$ is Majorana and of the same chirality as the supersymmetry parameter $\epsilon$. Hence, it admits a decomposition $\chi=\psi_{+} \otimes \eta_{+}-\psi_{-} \otimes \eta_{-}$with $\psi_{ \pm}$the 4-dimensional gaugino fields. Notice that this is not a zero-mode decomposition but the usual decomposition in terms of $S U(3)$ singlets. Then we obtain

$$
\Sigma_{m n p}=\frac{1}{16} \operatorname{Tr}\left(\bar{\chi} \Gamma_{m n p} \chi\right)=-\left(\psi_{-}^{\dagger} \psi_{+} \Omega_{m n p}+\psi_{+}^{\dagger} \psi_{-} \Omega_{m n p}^{*}\right)=-\left(\Lambda^{3} \Omega_{m n p}+\text { c.c. }\right),
$$

where $\Lambda^{3}=\frac{1}{2} \operatorname{Tr}\left(\bar{\psi}\left(1+\gamma_{(5)}\right) \psi\right)=\psi_{-}^{\dagger} \psi_{+}$the 4-dimensional condensate. We see that the condensate consists only of $(3,0)$ and $(0,3)$ pieces. This expansion is valid for $\Delta_{m n p}$ as well. By denoting $\delta^{3}=\frac{1}{2} \operatorname{Tr}\left(\bar{\lambda}\left(1+\gamma_{(5)}\right) \lambda\right.$ the vev of the 4-dimensional dilatino condensate, we have $\Delta_{m n p}=-\delta^{3} \Omega_{m n p}+$ c.c.

In order to expand correctly the dilatino-gaugino condensates $\Phi_{[\ldots]}$ we have to take into account that $\chi$ and $\lambda$ have opposite chiralities. Hence, $\lambda$ is expanded as $\lambda=\lambda_{+} \otimes \eta_{-}-\lambda_{-} \otimes \eta_{+}$ with $\lambda_{ \pm}$the 4 -dimensional dilatinos. Then we find that $\Phi$ is a real scalar $\Phi=-\left(\lambda_{+}^{\dagger} \chi_{-}+\right.$

\footnotetext{
${ }^{4}$ Our spinor conventions and some useful formulae can be found in Appendix A.

${ }^{5}$ As is common, we will use the same symbol for both the almost complex structure tensor and the associated 2-form.
} 
$\left.\lambda_{-}^{\dagger} \chi_{+}\right), \Phi_{m n}$ is a real 2-form $\Phi_{m n}=i\left(\lambda_{+}^{\dagger} \chi_{-}-\lambda_{-}^{\dagger} \chi_{+}\right) J_{m n} \equiv \Phi_{0} J_{m n}$, and $\Phi_{m n k l}$ is a real 4-form $\Phi_{m n k l}=-3 \Phi J_{[m n} J_{k l]}$. Also, all of them have an adjoint $E_{8} \times E_{8}$ index that we suppress.

The supercovariant H-flux (2.3) can be expanded in terms of the $S U(3)$-invariant forms as

$$
\hat{H}_{m n p}=\frac{1}{48} \Omega_{m n p} \hat{H}^{(3,0)}+\left(\hat{H}_{m n p}^{o(2,1)}+\frac{3}{4} \hat{H}_{[m}^{(1,0)} J_{n p]}\right)+c . c .,
$$

where $\hat{H}^{(3,0)}=\Omega^{* m n p} \hat{H}_{m n p}, \hat{H}_{m}^{(1,0)}=\left(\Pi^{+}\right)_{m}^{s} \hat{H}_{s n p} J^{n p}$ and $\hat{H}^{o(2,1)}$ the primitive $(2,1)$ piece of $\hat{H}_{m n p}$ which satisfies $\hat{H}^{o(2,1)} \wedge J=0$. Notice that due to (2.12) and the fact that $\Delta_{m n p}$ consists of only $(3,0)$ and $(0,3)$ pieces, we have $\hat{H}_{m n p}^{(2,1)}=H_{m n p}^{(2,1)}$.

Now, we are ready to proceed to the analysis of the supersymmetry variations (2.7), (2.8) and (2.9) for the setup under consideration.

\subsection{Conditions on the external geometry}

The 4-dimensional part of the gravitino variation reads as

$$
\delta \psi_{\mu}=\nabla_{\mu} \epsilon-\frac{1}{12} \tilde{\Sigma}_{m n p} \Gamma_{\mu} \Gamma^{m n p} \epsilon=0
$$

where we have introduced the combination $\tilde{\Sigma}_{m n p}=\Sigma_{m n p}+\frac{3}{4} \Delta_{m n p}=-\left(\tilde{\Lambda}^{3} \Omega_{m n p}+\right.$ c.c. $)$. Since $g_{M N}=e^{2(D-\phi)} \hat{g}_{M N}$ and $\Gamma_{M}=e^{(D-\phi)} \hat{\Gamma}_{M}$ we can rewrite eq. (3.9) as

$$
\delta \psi_{\mu}=\hat{\nabla}_{\mu} \epsilon+\frac{1}{2} \hat{\Gamma}_{\mu}{ }^{n} \hat{\nabla}_{n}(D-\phi) \epsilon-\frac{1}{12} e^{(D-\phi)} \hat{\Gamma}_{\mu} \tilde{\Sigma} \epsilon,
$$

and its integrability condition gives $\hat{\Gamma}^{\mu \nu} \hat{\nabla}_{\nu} \delta \psi_{\mu}=0[2]$.

By assumption the 4-dimensional metric $\hat{g}_{\mu \nu}(x)$ is maximally symmetric. Hence, the corresponding Riemann curvature tensor is of the form

$$
\hat{R}_{\nu \mu \kappa \lambda}=\frac{\hat{R}}{12}\left(\hat{g}_{\nu \kappa} \hat{g}_{\mu \lambda}-\hat{g}_{\nu \lambda} \hat{g}_{\mu \kappa}\right)
$$

with $\hat{R}$ the constant scalar curvature. For this metric we can show easily that $\hat{\Gamma}^{\mu \nu} \hat{\nabla}_{\nu} \hat{\nabla}_{\mu} \epsilon=$ $\frac{\hat{R}}{4} \epsilon$. Then the integrability condition yields

$$
\frac{\hat{R}}{4}-3 \hat{\nabla}^{n}(D-\phi) \hat{\nabla}_{n}(D-\phi)+3 \tilde{\Sigma}_{m} \hat{\nabla}_{m} e^{(D-\phi)}-\frac{1}{12} e^{2(D-\phi)} \tilde{\Sigma}^{2}=0
$$

For vanishing condensates eq. (3.11) reduces to eq. (2.8) of [2]. In this case one finds that $\hat{R}=0$ since the only possible constant value for $\hat{\nabla}^{n}(D-\phi) \hat{\nabla}_{n}(D-\phi)$ on a compact manifold is zero. Hence $M_{1,3}$ is Minkowski and the warp factor satisfies $D(y)=\phi(y)$ up to an additive constant. 
At first sight a non-zero condensate $\tilde{\Sigma}_{m n p}$ seems to open up a much wider range of possibilities. However, we will see in a moment that the supersymmetry conditions imply the constancy of $D-\phi$. Then $M_{1,3}$ is $A d S_{4}$ with constant negative curvature given by

$$
\hat{R}=\frac{1}{3} e^{2(D-\phi)} \tilde{\Sigma}^{2}
$$

Notice that $\tilde{\Sigma}^{2}$ is negative because $\tilde{\Sigma}$ is antihermitian.

Another way of computing the dependance of $\hat{R}$ on the vev of the condensate $\tilde{\Lambda}$ is the following. The 4-dimensional spinors in a maximally symmetric spacetime satisfy $\hat{\nabla}_{\mu} \theta_{+}=$ $W \hat{\gamma}_{\mu} \theta_{-}$and $\hat{\nabla}_{\mu} \theta_{-}=W^{*} \hat{\gamma}_{\mu} \theta_{+}$. The curvature is then $\hat{R}=-48|W|^{2}$. Using the fact that

$$
\nabla_{\mu} \theta_{+}=\hat{\nabla}_{\mu} \theta_{+}+\frac{1}{2} \Gamma_{\mu} \Gamma^{m} \partial_{m}(D-\phi) \theta_{+}=W e^{-(D-\phi)} \gamma_{\mu} \theta_{-}+\frac{1}{2} \gamma_{\mu} \gamma^{(5)} \theta_{+} \gamma^{m} \partial_{m}(D-\phi)
$$

and inserting the latter expression in (3.9) we obtain

$$
f W e^{-(D-\phi)} \eta_{+}+\frac{f^{*}}{2} \partial_{m}(D-\phi) \gamma^{m} \eta_{-}-\frac{f^{*}}{12} \tilde{\Sigma}_{m n p} \gamma^{m n p} \eta_{-}=0
$$

Multiplying now eq. (3.13) with $\eta_{+}^{\dagger}$ from the left we obtain

$$
f W e^{-(D-\phi)}-\frac{f^{*}}{12} \tilde{\Sigma}_{m n p}\left(\eta_{+}^{\dagger} \gamma^{m n p} \eta_{-}\right)=0 \Longrightarrow f W e^{-(D-\phi)}-\frac{f^{*}}{12}\left(\tilde{\Lambda}^{*}\right)^{3}\left\|\Omega^{*}\right\|^{2}=0,
$$

where $\|\Omega\|=\left(\Omega_{m n p}^{*} \Omega^{m n p}\right)^{1 / 2}$. Using Fierz identities one can find that $\|\Omega\|^{2}=48$. For vanishing total condensate $\tilde{\Lambda}^{3}=0$, we obtain $W=0$ and hence $M_{1,3}$ is flat. However in general it holds that

$$
\hat{R} \sim e^{2(D-\phi)}|\tilde{\Lambda}|^{6}
$$

Now we can prove the constancy of $D-\phi$. By multiplying eq. (3.13) with $\eta_{-}^{\dagger} \gamma_{n}$ from the left and using the equality $\eta_{-}^{\dagger} \gamma^{m n p q} \eta_{-}=-3 J^{[m n} J^{p q]}$ we obtain

$$
f^{*} \partial_{n}(D-\phi)-\frac{i}{2} f^{*} \partial_{m}(D-\phi) J_{n}^{m}-\frac{3}{256} f^{*} \tilde{\Sigma}^{m p q} J^{[m n} J^{p q]}+\frac{3 i}{256} f^{*} \tilde{\Sigma}_{n m p} J^{m p}=0 .
$$

Since $\tilde{\Sigma}$ is a $(3,0)+(0,3)$ form, we have $\tilde{\Sigma}_{n m p} J^{m p}=0$ and $\tilde{\Sigma}_{m p q} J^{[n m} J^{p q]}=0$. This immediately shows that

$$
\partial_{n}(D-\phi)=0
$$

and demonstrates that the constancy of $D-\phi$ is actually imposed upon us from the supersymmetry conditions. 


\subsection{Conditions on the internal geometry and intrinsic torsion classes}

\subsubsection{Gravitino variation}

Next we do the analysis of the 6-dimensional part of the gravitino variation. This reads as

$$
\delta \psi_{m}=\nabla_{m} \epsilon-\frac{1}{4}\left(\hat{H}_{m p q}-2 \tilde{\Sigma}_{m p q}+\frac{1}{6} \Delta_{m p q}\right) \Gamma^{p q} \epsilon-\frac{1}{12} \tilde{\Sigma}_{n p q} \Gamma_{m} \Gamma^{n p q} \epsilon=0 .
$$

Inserting the spinor ansatz (3.2) in eq. (3.18) we obtain

$$
\nabla_{m} \eta_{+}=-\partial_{m} \log f \eta_{+}+\frac{1}{4}\left(\hat{H}_{m p q}-2 \tilde{\Sigma}_{m p q}+\frac{1}{6} \Delta_{m p q}\right) \gamma^{p q} \eta_{+}+\frac{1}{12} \tilde{\Sigma}_{n p q} \gamma_{m} \gamma^{n p q} \eta_{+} .
$$

Using the identity

$$
\gamma^{m} \gamma^{n p q}=\gamma^{m n p q}+\delta^{m q} \gamma^{n p}+\delta^{m p} \gamma^{q n}+\delta^{m n} \gamma^{p q}
$$

we can rewrite eq. (3.19) as

$$
\nabla_{m} \eta_{+}=-\partial_{m} \log f \eta_{+}+\frac{1}{4}\left(\hat{H}_{m p q}-\tilde{\Sigma}_{m p q}+\frac{1}{6} \Delta_{m p q}\right) \gamma^{p q} \eta_{+}+\frac{1}{12} \tilde{\Sigma}_{n p q} \gamma_{m}{ }^{n p q} \eta_{+} \cdot
$$

Expressing $\gamma_{m}{ }^{n p q}$ as $\gamma_{m}{ }^{n p q}=-\frac{i}{2} \epsilon_{m}{ }^{n p q r s} \gamma_{r s} \gamma_{7}$ we then obtain

$$
\nabla_{m} \eta_{+}=-\partial_{m} \log f \eta_{+}+\frac{1}{4}\left(\hat{H}_{m p q}-\tilde{\Sigma}_{m p q}+\frac{1}{6} \Delta_{m p q}+i * \tilde{\Sigma}_{m p q}\right) \gamma^{p q} \eta_{+},
$$

where $* \tilde{\Sigma}_{m p q}=\frac{1}{3 !} \tilde{\Sigma}_{k l r} \epsilon^{k l r}{ }_{m p q}$.

However, the total contribution of $\tilde{\Sigma}_{m n p}$ in the above equations drops out. Indeed, due to the imaginary (anti)-self-duality of $\Omega_{m n p}\left(\Omega_{m n p}^{*}\right)$ we have $* \tilde{\Sigma}_{m p q}=-i \tilde{\Lambda}^{3} \Omega_{m p q}+$ c.c.. Using also the fact that $\Omega_{m p q} \gamma^{p q} \eta^{+}=0$, we finally obtain

$$
\nabla_{m} \eta_{+}=-\partial_{m} \log f \eta_{+}+\frac{1}{4} \widehat{H}_{m p q} \gamma^{p q} \eta_{+},
$$

where we have defined $\widehat{H}_{m n p}=\hat{H}_{m n p}+\frac{1}{6} \Delta_{m n p}$. Consequently, the spinor $\eta^{+}$is parallel under the connection with torsion $\nabla^{(-)}=\nabla-\frac{1}{4} \widehat{H}$. Defining the curvature through $\left[\nabla_{m}^{(-)}, \nabla_{n}^{(-)}\right] \eta^{+}=$ $\frac{1}{4} R_{m n p q}^{(-)} \gamma^{p q} \eta^{+}$, the integrability condition for eq. (3.22) implies

$$
R_{m n p q}^{(-)} J^{p q}=0
$$

Now using eq. (3.22) and its conjugate we find that $|f|=$ const. Hence, in general $f=e^{i \alpha}$. For our purposes, however, taking $f=1$ is not restrictive and we will do so.

It is straightforward to compute the covariant derivatives of the $S U(3)$-structure forms:

$$
\begin{aligned}
\nabla_{m} J_{n}{ }^{p} & =\left(\widehat{H}_{s m}^{p} J_{n}{ }^{s}+\widehat{H}_{m n}^{s} J_{s}{ }^{p}\right) \\
\nabla_{m} \Omega_{k l n} & =\widehat{H}_{m n}^{p} \Omega_{p k l}+\widehat{H}^{p}{ }_{l m} \Omega_{p k n}+\widehat{H}_{m k}^{p} \Omega_{p l n} .
\end{aligned}
$$

These relations imply that the internal space has contorsion given by $\widehat{H}_{m n p}$, which in turn has a contribution due to the H-flux and another due to the dilatino condensate. 


\subsubsection{Dilatino variation}

In the case that a non-zero gaugino condensate is present, the vanishing of the dilatino variation demands that $\hat{H}_{m n p}$ has a non-zero $(3,0)+(0,3)$ piece. Indeed, the dilatino variation is

$$
\delta \lambda=\Gamma^{m} \nabla_{m} \phi \epsilon+\frac{1}{24}\left(\hat{H}_{m n p}+\Sigma_{m n p}\right) \Gamma^{m n p} \epsilon=0,
$$

and for the spinor ansatz (3.2) it becomes

$$
\partial_{m} \phi \gamma^{m} \eta_{+}+\frac{1}{24}\left(\hat{H}_{m n p}+\Sigma_{m n p}\right) \gamma^{m n p} \eta_{+}=0
$$

Multiplying eq. (3.27) by $\eta_{-}^{\dagger}$ from the left gives

$$
\left(\hat{H}_{m n p}+\Sigma_{m n p}\right) \Omega^{m n p}=0
$$

This fixes the $(3,0)$ and $(0,3)$ parts of the supercovariantized flux in terms of the gaugino condensate vev

$$
\hat{H}_{m n p}^{(3,0)}=\Lambda^{3} \Omega_{m n p} .
$$

If the dilatino condensate is zero, so that $\hat{H}_{m n p}=H_{m n p}$, the above equations imply that in order to preserve supersymmetry in the presence of a non-vanishing gaugino condensate an H-flux with non-vanishing $(3,0)$ and $(0,3)$ components should be present too.

Multiplying now eq. (3.27) by $\eta_{+}^{\dagger} \gamma_{k}$ from the left gives

$$
i J_{k}^{n} \partial_{n} \phi+\partial_{k} \phi-\frac{1}{8} \hat{H}_{m n p} J_{k}^{m} J^{n p}+\frac{i}{8} \hat{H}^{k m n} J^{m n}=0 \text {. }
$$

The contribution of $\Sigma_{m n p}$ drops out since the gaugino condensate has only $(3,0)+(0,3)$ components. From eq. (3.30) we obtain an equation for the dependance of the dilaton on the internal space coordinates

$$
\partial_{k} \phi=\frac{1}{8} \hat{H}_{m n p} J_{k}^{m} J^{n p}=\frac{i}{8}\left(H^{(1,0)}-H^{(0,1)}\right) .
$$

We see that only the non-primitive $(2,1)+(1,2)$ piece of $\hat{H}_{m n p}$ (and hence of $H_{m n p}$ since they only differ in their $(3,0)+(0,3)$ parts $)$, contributes to the variation of the dilaton. In particular, if we have only non-zero the $(3,0)$ and $(0,3)$ components of the H-flux, the dilaton is constant, $\partial_{k} \phi=0$. Then due to eq. (3.17) the warp factor $D(y)$ is constant too. This shows that the assumptions of refs. [21, 22] on the constancy of $D$ and $\phi$ are not just sufficient but also necessary for unbroken supersymmetry. 


\subsubsection{Gaugino variation}

Finally we consider the gaugino variation. This is given by

$$
\delta \chi=-\frac{1}{4} \hat{F}_{m n} \Gamma^{m n} \epsilon+\frac{1}{32}\left(3 \Phi-\frac{3}{2} \Phi_{m n} \Gamma^{m n}-\frac{1}{24} \Phi_{m n k l} \Gamma^{m n k l}\right) \epsilon,
$$

while inserting the spinor ansatz and the decomposition of the condensates yields

$$
\delta \chi=-\frac{1}{4}\left(F_{m n}+\frac{3}{16} \Phi_{0} J_{m n}\right) \gamma^{m n} \eta_{+}
$$

where the terms proportional to $\Phi$ are zero due to the identity $\left(1+\frac{1}{24} J_{m n} J_{k l} \gamma^{m n k l}\right) \eta_{+}=0$.

A vanishing gaugino variation demands that the field strength satisfies $F_{m n}=J_{m}^{k} J_{n}{ }^{l} F_{k l}$, i.e. $F^{(2,0)}=F^{(0,2)}=0$. Furthermore, the non-primitive part of $F_{m n}$ is compensated by the vev of the dilatino-gaugino condensate $\Phi_{0}$ as $\Phi_{0}=-\frac{8}{9} F_{m n} J^{m n}$. In the usual case where this condensate is taken to be zero, we end up with the standard result that a supersymmetry preserving background gauge field has to be a primitive $(1,1)$ form.

\subsection{Torsion classes}

We can summarize now our findings in the language of intrinsic torsion classes which are defined as

$$
d J=\frac{3 i}{4}\left(\mathcal{W}_{1} \Omega^{*}-\overline{\mathcal{W}}_{1} \Omega\right)+\mathcal{W}_{4} \wedge J+\mathcal{W}_{3}
$$

and

$$
d \Omega=\mathcal{W}_{1} J \wedge J+\mathcal{W}_{2} \wedge J+\mathcal{W}_{5}^{*} \wedge \Omega
$$

satisfying $J \wedge \mathcal{W}_{3}=J \wedge J \wedge \mathcal{W}_{2}=\Omega \wedge \mathcal{W}_{3}=0$. The classes $\mathcal{W}_{1}$ and $\mathcal{W}_{2}$ can be decomposed in real and imaginary parts as $\mathcal{W}_{1}=\mathcal{W}_{1}^{+}+\mathcal{W}_{1}^{-}$and $\mathcal{W}_{2}=\mathcal{W}_{2}^{+}+\mathcal{W}_{2}^{-}$.

The classes $\mathcal{W}_{1}$ and $\mathcal{W}_{2}$ are vanishing when the almost complex structure is integrable, i.e. when the manifold is complex. A Kähler manifold has furthermore $\mathcal{W}_{3}=\mathcal{W}_{4}=0$. Finally, CY manifolds have in addition $\mathcal{W}_{5}=0$. Hence, one can think of the five torsion classes as parameterizing the deformation away from $S U(3)$-holonomy.

Using eqs. (3.24) and (3.25) we obtain

$$
\begin{aligned}
& \mathcal{W}_{1}=\frac{1}{6} \widehat{H}^{(0,3)}=8\left(\left(\Lambda^{*}\right)^{3}-\frac{1}{6}\left(\delta^{*}\right)^{3}\right), \\
& \mathcal{W}_{2}=0 \\
& \mathcal{W}_{3}=i\left(H^{o(2,1)}-H^{o(1,2)}\right), \\
& \mathcal{W}_{4}=-\frac{i}{2}\left(H^{(1,0)}-H^{(0,1)}\right), \\
& \mathcal{W}_{5}=i\left(H^{(1,0)}-H^{(0,1)}\right) .
\end{aligned}
$$


In order to derive the class $\mathcal{W}_{1}$, we took into account eq. (3.29) and the definition $\widehat{H}_{m n p}=$ $\hat{H}_{m n p}+\frac{1}{6} \Delta_{m n p}$. Using eq. (3.31) we can express the torsion classes $\mathcal{W}_{4}$ and $\mathcal{W}_{5}$ in terms of the dilaton gradient as

$$
\mathcal{W}_{4}=-4 d \phi, \quad \mathcal{W}_{5}=8 d \phi .
$$

Now we summarize our findings in order to be easy to contrast them to the case with $\mathrm{H}$ flux but without condensates [6] and to the case with H-flux and only the gaugino condensate [18. We have found that:

- The gaugino condensate $\Sigma_{m n p}$ induces a $(3,0)+(0,3)$ piece to the supercovariant $\hat{H}$ flux.

- The dilatino and gaugino condensate yield a non-vanishing $\mathcal{W}_{1}$ class, rendering the internal spaces non-complex.

- The class $\mathcal{W}_{2}$ is zero for the H-flux and for all the condensates we turned on.

- The intrinsic torsion classes $\mathcal{W}_{i}, i=3,4,5$ are determined only in terms of the $(2,1)+$ $(1,2)$ pieces of the flux and hence do not depend on the condensates.

- The spacetime curvature depends on the dilatino and gaugino condensates in such a way that one can tune them to obtain a Minkowski vacuum with a non-complex internal space ${ }^{6}$.

- The dilatino-gaugino condensates in the gaugino variation allow for background gauge fields that can have a non-primitive $(1,1)$ piece.

\section{Supersymmetric solutions on nearly-Kähler spaces}

In the previous section we presented a set of conditions on the intrinsic torsion classes of a six-dimensional manifold with $S U(3)$-structure, which are necessary for obtaining supersymmetric vacua of heterotic string theory in the presence of $\mathrm{H}$-flux and several condensates. The most general manifolds with torsion classes specified by eq. (3.36) are known as $\mathcal{G}_{1}$ manifolds [16].

In the absence of condensates, only an H-flux with non-trivial $(2,1)+(1,2)$ components can be present if some supersymmetry is to be preserved. In particular, if only the primitive part of the flux is non-zero we have $\mathcal{W}_{1}=\mathcal{W}_{2}=\mathcal{W}_{4}=\mathcal{W}_{5}=0$. These manifolds are known as special-hermitian and are well-studied in the mathematical literature. However, so far it

\footnotetext{
${ }^{6} \mathrm{~A}$ similar conclusion was reached in ref. 26] for compactifications of 11-dimensional supergravity with gravitino condensates.
} 
has been proved that is difficult to satisfy the Bianchi identity for compactifications of this type.

Another natural choice is to assume that only the condensates and the corresponding pieces of the H-flux induced by them are non-vanishing. In this case, the H-flux is of $(3,0)+$ $(0,3)$ type and, according to eq. (3.36), the appropriate six-dimensional manifolds will have only the class $\mathcal{W}_{1}$ different from zero. Notice that supersymmetry specifies further the H-flux in terms of the condensates, effectively determining $\mathcal{W}_{1}$ in terms of $\Lambda^{3}$ and $\delta^{3}$. Setups with $\delta^{3}=0$ have been already considered in refs. [21, 22. One of our purposes here is to revisit them in the framework of $S U(3)$-structures and intrinsic torsion. Besides that, the analysis of the previous section showed that some of the assumptions of refs. 21, 22] are actually necessary and not just sufficient for preserving spacetime supersymmetry. Furthermore, the authors of refs. 21, 22 did not take into account the modifications to the hererotic Bianchi identities due to the non-trivial torsion. As we shall see, to satisfy the Bianchi identity, the presence of the gaugino condensate is not enough.

Six-dimensional manifolds with non-zero $\mathcal{W}_{1}$ have been well-studied too in the mathematical literature. They are known as nearly-Kähler and they have certain special properties. Among others, they are Einstein spaces of positive scalar curvature, their almost complex structure is never integrable since the Nijenhuis tensor is non-zero, their first Chern class vanishes, and they admit a spin structure. In the ensuing we will discuss in detail some examples of nearly-Kähler spaces which are based on coset spaces.

Let us just mention for the moment that it would be interesting to relax the condition $H^{(2,1)}=0$ and turn-on a primitive $(2,1)+(1,2)$ piece of the $\mathrm{H}$-flux (in addition to the $(0,3)+(3,0)$ piece induced by the condensates $)$. Then the conditions on the intrinsic torsion would imply that the compactification manifold should have $\mathcal{W}_{2}=\mathcal{W}_{4}=\mathcal{W}_{5}=0$ and (choosing purely imaginary condensates) $\mathcal{W}_{1}^{+}=0$. Such manifolds are particular cases of half-flat manifolds and one simple but interesting realization is provided by twisted toroidal orbifolds 42] (see also [43] for the 7-dimensional analogue). It would be worthwhile to investigate if more general solutions can be provided by such spaces, but we postpone that for future work.

\subsection{Nearly-Kähler coset spaces}

The only known examples of compact nearly-Kähler spaces are 3-symmetric spaces that can be described as homogeneous cosets. These are (i) $G_{2} / S U(3)$, which is an $S^{6}$ but less symmetric than the usual 6 -sphere, (ii) $S p(4) /(S U(2) \times U(1))_{\text {non-max. }}$ which is similarly a less symmetric version of $\mathbb{C P}_{3}$, (iii) $S U(3) /(U(1) \times U(1))$ which is the flag manifold $F(1,2)$, and (iv) $S U(2)^{3} / S U(2)$ which is isomorphic to $S^{3} \times S^{3}$. All these spaces admit an $S U(3)$ - 
structure. Although in general they are half-flat, there are special values of their moduli for which only $\mathcal{W}_{1}^{-}$is non-zero. For these special values these cosets become nearly-Kähler.

In Appendix B we present the $S U(3)$-structures of the 4 cosets and compute their intrinsic torsion classes. Inspection of the results shows that all possible radii of the cosets have to be the same if we want to keep a vanishing $\mathcal{W}_{2}$ class. Then, the only non-zero class is $\mathcal{W}_{1}$ and it takes the general form $\mathcal{W}_{1}=-i \frac{w}{\sqrt{a}}$. The actual values of $w$ for each coset can be found in Appendix B.

Furthermore, using the corresponding curvature 2-forms [55], we can compute the first Pontrjagin classes:

$$
\begin{aligned}
G_{2} / S U(3) & : \operatorname{tr} R \wedge R=0, \\
S p(4) /(S U(2) \times U(1)) & : \operatorname{tr} R \wedge R=\frac{18}{a^{2}} J \wedge J, \\
S U(3) /(U(1) \times U(1)) & : \operatorname{tr} R \wedge R=\frac{18}{a^{2}} J \wedge J, \\
S U(2)^{3} / S U(2) & : \operatorname{tr} R \wedge R=-\left(\frac{8}{9 a}\right)^{2} J \wedge J .
\end{aligned}
$$

Hence, for all cosets under consideration and for metrics with nearly-Kähler structure we have a general formula for the first Pontrjagin class given by $\operatorname{tr} R \wedge R=\frac{p_{1}}{a^{2}} J \wedge J$. Since $J \wedge J$ is exact, this class is cohomologically trivial. Using the general expression for $\mathcal{W}_{1}$ we can also write

$$
\operatorname{tr} R \wedge R=\frac{p_{1}}{w^{4}}\left|\mathcal{W}_{1}\right|^{4} J \wedge J
$$

The supersymmetry conditions demand that the torsion class $\mathcal{W}_{1}$ is fixed in terms of the condensates. This stabilizes the radial modulus to a value

$$
a=-\frac{w^{2}}{8}\left|\Lambda^{3}-\frac{1}{6} \delta^{3}\right|^{-2}
$$

Since $\mathcal{W}_{1}$ is imaginary for the cosets under consideration, the condensate combination $\left(\Lambda^{*}\right)^{3}-$ $\frac{1}{6}\left(\delta^{*}\right)^{3}$ has to be imaginary too.

\subsection{Bianchi identity}

Let us now consider the Bianchi identity for the H-flux. The full Bianchi identity includes a correction due to the gravitational Chern-Simons term required for anomaly cancelation [44] and it reads

$$
d H=\frac{1}{2}\left(\operatorname{tr} R^{(+)} \wedge R^{(+)}-\frac{1}{30} \operatorname{Tr} F \wedge F\right)
$$

where $R^{(+)}$is the curvature 2 -form of the connection $\nabla^{(+)}=\nabla+\frac{1}{4} \widehat{H}$. We have also adopted the usual normalization for the trace of the gauge field strengths. 
The torsion of the connection whose curvature appears in the Bianchi identity is the opposite of the torsion of the connection $\nabla^{(-)}=\nabla-\frac{1}{4} \widehat{H}$ appearing in the supersymmetry variation of the gravitino in the internal space. Although this fact is well-established only for the case where the torsion comes entirely from the H-flux [45], it is quite natural to expect that the proper generalization of the gravitational Chern-Simons correction to the Bianchi involves the full torsion tensor appearing in the gravitino variation. Let us also mention that demanding only supersymmetry and anomaly freedom does not specify completely the torsion relevant for the gravitational Chern-Simons term [46]. From this point of view our choice is perfectly consistent. Notice, furthermore, that the ambiguity is fixed by the additional requirement of conformal invariance on the worldsheet [45] or, equivalently, of satisfying the spacetime equations of motion. It would be extremely interesting and important to actually verify the validity of our choice with an explicit calculation. However, such a computation is bound to be subtle due to the presence of the condensates.

We can now proceed with the computation of the quantities that appear in the Bianchi identity. The curvature tensor for $\nabla^{(+)}=\nabla+\frac{1}{4} \widehat{H}$ is

$$
R_{m n p q}^{(+)}=R_{m n p q}+2 \nabla_{[m} \widehat{H}_{n] p q}-\left(\widehat{H}_{m p}{ }^{r} \widehat{H}_{r n q}-\widehat{H}_{n p}{ }^{r} \widehat{H}_{r m q}\right)
$$

and the corresponding curvature 2 -form is

$$
R_{m n}^{(+)}=\frac{1}{2} R_{m n p q}^{(+)} e^{p} \wedge e^{q} .
$$

For nearly-Kähler spaces the torsion $\widehat{H}_{m n p}$ has only $(3,0)+(0,3)$ parts which are fixed by the class $\mathcal{W}_{1}$ as

$$
\widehat{H}_{m p q}=8\left(\mathcal{W}_{1} \Omega_{m p q}+\text { c.c. }\right) .
$$

Using eq. (3.25) and the identity

$$
\Omega_{p m n} \Omega^{* p k l}=16\left(\Pi^{+}\right)_{[m}^{[k}\left(\Pi^{+}\right)_{n]}^{l]}
$$

(c.f. for instance [12]), we obtain

$$
\begin{aligned}
\nabla_{[m} \widehat{H}_{n] p q} & =24\left|\mathcal{W}_{1}\right|^{2} J_{[m n} J_{p q]} \\
\widehat{H}_{m p}{ }^{r} \widehat{H}_{r n q}-\widehat{H}_{n p}^{r} \widehat{H}_{r m q} & =4\left|\mathcal{W}_{1}\right|^{2}\left(\left(g_{m p} g_{n q}-g_{m q} g_{n p}\right)-J_{m n} J_{p q}-3 J_{[m n} J_{p q]}\right) .
\end{aligned}
$$

Subsequently, the generalized curvatures are found to be

$$
\begin{aligned}
& R_{m n p q}^{(+)}=R_{m n p q}+60\left|\mathcal{W}_{1}\right|^{2} J_{[m n} J_{p q]}-4\left|\mathcal{W}_{1}\right|^{2}\left(\left(g_{m p} g_{n q}-g_{m q} g_{n p}\right)-J_{m n} J_{p q}\right), \\
& R_{m n p q}^{(-)}=R_{m n p q}-36\left|\mathcal{W}_{1}\right|^{2} J_{[m n} J_{p q]}-4\left|\mathcal{W}_{1}\right|^{2}\left(\left(g_{m p} g_{n q}-g_{m q} g_{n p}\right)-J_{m n} J_{p q}\right) .
\end{aligned}
$$


The left-hand-side of the Bianchi identity (4.4) can be derived by using the fact that the $\hat{H}_{m n p} \equiv H_{m n p}-6 \Delta_{m n p}$ is fixed, by the vanishing of the dilatino variation, to be $\hat{H}_{m n p}=$ $\Lambda^{3} \Omega_{m n p}+$ c.c.. Since for nearly-Kähler spaces holds that $d \Omega=\mathcal{W}_{1} J \wedge J$, we find that

$$
d H=\left(\Lambda^{3}-6 \delta^{3}\right) \mathcal{W}_{1} J \wedge J+\text { c.c. }
$$

Then we can eliminate the explicit dependance on $\Lambda$ and its conjugate by using the supersymmetry condition $\mathcal{W}_{1}=8\left(\left(\Lambda^{*}\right)^{3}-\frac{1}{6}\left(\delta^{*}\right)^{3}\right)$. Finally we obtain

$$
d H=\left(\frac{1}{4}\left|\mathcal{W}_{1}\right|^{2}-\operatorname{Re}\left(\delta^{3} \mathcal{W}_{1}\right)\right) J \wedge J
$$

where we have rescaled $\delta^{3} \rightarrow \frac{18}{35} \delta^{3}$.

The right-hand side of the Bianchi identity (4.4) depends on the first Pontrjagin class of $\nabla^{(+)}$. After a slightly tedious calculation we obtain

$$
\operatorname{tr} R^{(+)} \wedge R^{(+)}=\operatorname{tr} R \wedge R-4608\left|\mathcal{W}_{1}\right|^{4} J \wedge J
$$

Next, to obtain a feeling of the implications of the Bianchi identity (4.13), we assume that there is no background gauge field. Using eq. (4.2) we find that the Bianchi identity reduces to the following equation

$$
\left(\frac{1}{4}\left|\mathcal{W}_{1}\right|^{2}-\operatorname{Re}\left(\delta^{3} \mathcal{W}_{1}\right)\right)=\left(\frac{p_{1}}{2 w^{4}}-2304\right)\left|\mathcal{W}_{1}\right|^{4}
$$

In addition, for simplicity let us consider the case $G_{2} / S U(3)$ for which $p_{1}=0$. Recall that for all cosets the class $\mathcal{W}_{1}$ is imaginary. Then we find the following solution for $\delta^{3}$, which in the present case is purely imaginary:

$$
\delta^{3}=-i\left|\mathcal{W}_{1}\right|\left(\frac{1}{4}+2304\left|\mathcal{W}_{1}\right|^{2}\right)
$$

Moreover, the gaugino condensate is fixed to a purely imaginary value as well given by

$$
\operatorname{Im}\left(\Lambda^{3}\right)=\frac{3}{35} \operatorname{Im}\left(\delta^{3}\right)-\frac{1}{8}\left|\mathcal{W}_{1}\right|
$$

which in terms of the class $\mathcal{W}_{1}$ becomes

$$
\operatorname{Im}\left(\Lambda^{3}\right)=-\frac{1}{35}\left|\mathcal{W}_{1}\right|\left(\frac{41}{8}+3 \cdot 2304\left|\mathcal{W}_{1}\right|^{2}\right)
$$

It is straightforward to find similar solutions for the other three cosets.

It is clear that the previous solution retains in four dimensions the full gauge group $E_{8} \times E_{8}$ and thus it is undesirable as a realistic GUT. However, we can turn on a background gauge field that breaks part of the gauge symmetry in a supersymmetric way. The condition for 
supersymmetry dictates that the gaugino variation (3.33) is zero. A simple way to deal with this is to assume that all the condensates $\Phi_{[\ldots]}$ are vanishing and to consider further the curvature 2-form corresponding to the $S U(3)$ connection with torsion $\nabla^{(-)}$. Embedding this connection in the gauge group, yields a background $S U(3)$ gauge field $\left(F_{a b}\right)_{m n}=R_{a b m n}^{(-)}$ which leads to a vanishing gaugino variation as a consequence of the integrability condition $R_{a b m n}^{(-)} \gamma^{m n} \eta^{+}=0$. For such a choice $\frac{1}{30} \operatorname{Tr} F \wedge F=\operatorname{tr} R^{(-)} \wedge R^{(-)}$, where the latter is found to be

$$
\operatorname{tr} R^{(-)} \wedge R^{(-)}=\operatorname{tr} R \wedge R+1536\left|\mathcal{W}_{1}\right|^{4} J \wedge J
$$

The Bianchi identity leads now to the relation

$$
\left(\frac{1}{4}\left|\mathcal{W}_{1}\right|^{2}-\operatorname{Re}\left(\delta^{3} \mathcal{W}_{1}\right)\right)=-3072\left|\mathcal{W}_{1}\right|^{4}
$$

which fixes the dilatino condensate and, in turn, also the gaugino condensate by the supersymmetry condition (4.3). The background gauge field breaks the gauge group down to $E_{6} \times E_{8}$ as does the standard embedding in the case of CY compactifications.

Using now the fact that $\Omega_{m n p}\left(\Omega_{m n p}^{*}\right)$ is imaginary (anti)-self-dual, one can easily check that for all the above solutions the NS-NS flux is coclosed:

$$
d * H=0
$$

and hence it solves the source-free equation of motion. Accordingly, since the Bianchi equation and the equation of motion for $H$ are satisfied and the background preserves some supersymmetry, the Einstein equations are also verified as a consequence of the results of [27.

\section{Conclusions}

In the present work we have examined in detail some examples of supersymmetric AdS compactifications of heterotic strings on non-complex manifolds, which we believe will be eventually phenomenologically interesting. The considered six-dimensional manifolds include the coset spaces $G_{2} / S U(3), S p(4) /(S U(2) \times U(1))_{\text {non-max }}, S U(3) /(U(1) \times U(1))$ and $S U(2)^{3} / S U(2)$. These have been examined some time ago in the context of Coset Space Dimensional Reduction [47, 48, 49, in attempts to obtain realistic GUTs from extra dimensions [31, 32, 33, 50, 51], and also in the context of heterotic sting theory in studies of possible supersymmetry preserving backgrounds in refs. [19, 20, 21, 22]. These solutions have been analyzed here in the framework of $G$-structures while a new example based on the coset $S U(2)^{3} / S U(2)$ has been added. 
The conjecture that nearly-Kähler spaces have no complex-structure moduli [52] and the fact that the supersymmetry preserving conditions fix the radii to specific values, implies that one should expect supersymmetric AdS vacua with most moduli stabilized in this approach. Subsequently, it would be worthwhile to analyze the possibilities for "lifting" the AdS vacuum to Minkowski or de Sitter [28, 30] and study supersymmetry breaking in this context. Work in this direction is currently in progress.

\section{Acknowledgments}

We would like to thank K. Behrndt, G.L. Cardoso, G. Dall'Agata, J.-P. Derendinger, A. Frey, P. Forgacs, J. Gauntlett, M. Lippert, D. Lüst, A. Micu, A. Nagy, G. Papadopoulos, K. Sfetsos, A. Uranga and D. Zoakos for helpful discussions and correspondence. We are also grateful to A. Frey, M. Lippert and D. Tsimpis for interesting comments and questions on the first version of this paper, as well as to T. Kimura for pointing out a numerical mistake.

The work is supported by the EPEAEK programme "Pythagoras" and co-founded by the European Union (75\%) and the Hellenic state (25\%). The work of N.P. is supported by the Swiss National Science Foundation and by the Commission of the European Communities under contract MRTN-CT-2004-005104.

\section{Appendix A: Gamma matrix conventions}

The 10-dimensional Gamma matrices $\Gamma^{M}$ with $M=0,1, \ldots, 9$ satisfy $\left\{\Gamma^{M}, \Gamma^{N}\right\}=2 \eta^{M N}$ where $\eta^{M N}=(-1,1, \ldots, 1)$. Splitting to $4+6$ indices $\mu, \nu=0,1,2,3$ and $m, n=4, \ldots, 9$ we can write them as $\Gamma^{\mu}=\gamma^{\mu} \otimes \mathbb{I}$ and $\Gamma^{m}=\gamma^{5} \otimes \gamma^{m}$ where $\gamma^{\mu}$ the usual 4-dimensional gamma matrices, $\gamma^{(5)}$ is the chirality matrix satisfying $\left(\gamma^{(5)}\right)^{2}=1$ and $\left\{\gamma^{\mu}, \gamma^{(5)}\right\}=0$, and $\gamma^{m}$ are the Gamma matrices in 6-dimensional Euclidean space.

The 6-dimensional spinors $\eta_{ \pm}$have opposite chiralities $\gamma_{(7)} \eta_{ \pm}= \pm \eta_{ \pm}$where $\gamma_{(7)}=$ $-i \gamma_{4} \ldots \gamma_{9}$. They satisfy $\eta_{ \pm}^{\dagger}=\eta_{\mp}^{T} C_{(6)}$ where $C_{(6)}$ is the 6-dimensional charge conjugation matrix. The 4-dimensional Weyl spinors $\theta_{ \pm}$are also chiral $\gamma_{(5)} \theta_{ \pm}= \pm \theta_{ \pm}$with $\gamma_{(5)}=i \gamma_{0} \gamma_{1} \gamma_{2} \gamma_{3}$. Moreover $\bar{\theta}_{ \pm}= \pm \theta_{\mp}^{T} C_{(4)}$ with $C_{(4)}$ the 4-dimensional charge conjugation matrix. As usual, for a Dirac spinor $\psi$ we define $\bar{\psi}=\psi^{\dagger} \gamma^{0}$.

The 10-dimensional chirality and charge conjugation matrix are $\Gamma_{(11)}=\gamma_{(5)} \otimes \gamma_{(7)}$ and $C_{(10)}=C_{(4)} \gamma_{(5)} \otimes C_{(6)}$. With these definitions the spinor (3.2) satisfies $\Gamma_{(11)} \epsilon=\epsilon$ and $\bar{\epsilon}=\epsilon^{T} C_{(10)}$, i.e. it is chiral and Majorana. For the gamma matrix manipulations we used 53 . 
Finally we present some spinor bilinears that were used in the considerations of section 3:

$$
\begin{aligned}
\eta_{+}^{\dagger} \eta_{+} & =\eta_{-}^{\dagger} \eta_{-}=1, \\
\eta_{-}^{\dagger} \eta_{+} & =\eta_{+}^{\dagger} \eta_{-}=0 \\
\eta_{ \pm}^{\dagger} \gamma^{m} \eta_{ \pm} & =\eta_{\mp}^{\dagger} \gamma^{m} \eta_{ \pm}=0 \\
\eta_{ \pm}^{\dagger} \gamma^{m n} \eta_{\mp} & =\eta_{ \pm}^{\dagger} \gamma^{m n p q} \eta_{\mp}=0 \\
\eta_{ \pm}^{\dagger} \gamma^{m n} \eta_{ \pm} & = \pm i J^{m n} \\
\eta_{ \pm}^{\dagger} \gamma^{m n p q} \eta_{ \pm} & =-3 J^{[m n} J^{p q]} \\
\eta_{ \pm}^{\dagger} \gamma^{m n p} \eta_{ \pm} & =\eta_{\mp}^{\dagger} \gamma^{m n p q r s} \eta_{ \pm}=0 \\
\eta_{ \pm}^{\dagger} \gamma^{m n p q r} \eta_{ \pm} & =\eta_{\mp}^{\dagger} \gamma^{m n p q r} \eta_{ \pm}=0
\end{aligned}
$$

\section{Appendix B: $S U(3)$-structures on coset spaces}

In 54] the intrinsic torsion classes for the coset $S U(3) /(U(1) \times U(1))$ were calculated in terms of the radii $a, b, c$. Here we extend this computation to the rest of the coset spaces that admit a nearly-Kähler structure. The data required for this exercise can be found in [55, 56.

$S U(3) /(U(1) \times U(1))$

- Metric:

$$
d s^{2}=a\left(e^{1} \otimes e^{1}+e^{2} \otimes e^{2}\right)+b\left(e^{3} \otimes e^{3}+e^{4} \otimes e^{4}\right)+c\left(e^{5} \otimes e^{5}+e^{6} \otimes e^{6}\right) .
$$

- $S U(3)$-structure:

$$
\begin{aligned}
J & =-a e^{1} \wedge e^{2}+b e^{3} \wedge e^{4}-c e^{5} \wedge e^{6} \\
d J & =-(a+b+c)\left(e^{1} \wedge e^{3} \wedge e^{5}+e^{1} \wedge e^{4} \wedge e^{6}-e^{2} \wedge e^{3} \wedge e^{6}+e^{2} \wedge e^{4} \wedge e^{5}\right), \\
\Omega & =\sqrt{(a b c)}\left(e^{1}+i e^{2}\right) \wedge\left(e^{3}-i e^{4}\right) \wedge\left(e^{5}+i e^{6}\right) \\
d \Omega & =4 i \sqrt{a b c}\left(e^{1} \wedge e^{2} \wedge e^{3} \wedge e^{4}-e^{1} \wedge e^{2} \wedge e^{5} \wedge e^{6}+e^{3} \wedge e^{4} \wedge e^{5} \wedge e^{6}\right) .
\end{aligned}
$$

- Non-vanishing intrinsic torsion classes:

$$
\begin{aligned}
& \mathcal{W}_{1}=-\frac{2 i}{3} \frac{a+b+c}{\sqrt{a b c}} \\
& \mathcal{W}_{2}=-\frac{4 i}{3} \frac{1}{\sqrt{a b c}}\left[a(2 a-b-c) e^{12}-b(2 b-a-c) e^{34}+c(2 c-a-b) e^{56}\right] .
\end{aligned}
$$


$S U(2) \times S U(2)$

- Metric:

$$
d s^{2}=a\left(\frac{1}{3} e^{1} \otimes e^{1}+3 e^{6} \otimes e^{6}\right)+b\left(\frac{1}{3} e^{2} \otimes e^{2}+3 e^{5} \otimes e^{5}\right)+c\left(\frac{1}{3} e^{3} \otimes e^{3}+3 e^{4} \otimes e^{4}\right) .
$$

- $S U(3)$-structure:

$$
\begin{aligned}
J & =a e^{1} \wedge e^{6}-b e^{2} \wedge e^{5}+c e^{3} \wedge e^{4}, \\
d J & =-\frac{1}{\sqrt{3}}(a+b-c) e^{1} \wedge e^{2} \wedge e^{4}-\frac{1}{\sqrt{3}}(a-b+c) e^{1} \wedge e^{3} \wedge e^{5} \\
& +\frac{1}{\sqrt{3}}(a-b-c) e^{2} \wedge e^{3} \wedge e^{6}+\sqrt{3}(a+b+c) e^{4} \wedge e^{5} \wedge e^{6}, \\
\Omega & =i \sqrt{\frac{a b c}{27}}\left(e^{1}-3 i e^{6}\right) \wedge\left(e^{2}+3 i e^{5}\right) \wedge\left(e^{3}-3 i e^{4}\right), \\
d \Omega & =\frac{4 i}{3} \sqrt{a b c}\left(e^{1} \wedge e^{2} \wedge e^{5} \wedge e^{6}-e^{1} \wedge e^{3} \wedge e^{4} \wedge e^{6}+e^{2} \wedge e^{3} \wedge e^{4} \wedge e^{5}\right) .
\end{aligned}
$$

- Non-vanishing intrinsic torsion classes:

$$
\begin{aligned}
\mathcal{W}_{1} & =-\frac{2 i}{9 \sqrt{a b c}}(a+b+c), \\
\mathcal{W}_{2} & =\frac{4 i}{27 \sqrt{a b c}}\left(-a(2 a-b-c) e^{1} \wedge e^{6}+b(2 b-a-c) e^{2} \wedge e^{5}\right. \\
& \left.-c(2 c-a-b) e^{3} \wedge e^{4}\right), \\
\mathcal{W}_{3} & =-\frac{2}{3 \sqrt{3}}\left((a+b-2 c) e^{1} \wedge e^{2} \wedge e^{4}+(a-2 b+c) e^{1} \wedge e^{3} \wedge e^{5}\right. \\
& \left.+(-2 a+b+c) e^{2} \wedge e^{3} \wedge e^{6}\right) .
\end{aligned}
$$

$S p(4) /(S U(2) \times U(1))_{n o n-\max }$

- Metric:

$$
d s^{2}=a\left(e^{1} \otimes e^{1}+e^{2} \otimes e^{2}\right)+b\left(e^{3} \otimes e^{3}+e^{4} \otimes e^{4}\right)+a\left(e^{5} \otimes e^{5}+e^{6} \otimes e^{6}\right) .
$$

- $S U(3)$-structure:

$$
\begin{aligned}
J & =-a e^{1} \wedge e^{2}+b e^{3} \wedge e^{4}-a e^{5} \wedge e^{6}, \\
d J & =-(2 a+b)\left(e^{1} \wedge e^{3} \wedge e^{5}+e^{1} \wedge e^{4} \wedge e^{6}-e^{2} \wedge e^{3} \wedge e^{6}+e^{2} \wedge e^{4} \wedge e^{5}\right), \\
\Omega & =\sqrt{a^{2} b}\left(e^{1}+i e^{2}\right) \wedge\left(e^{3}-i e^{4}\right) \wedge\left(e^{5}+i e^{6}\right), \\
d \Omega & =4 i \sqrt{a^{2} b}\left(e^{1} \wedge e^{2} \wedge e^{3} \wedge e^{4}-e^{1} \wedge e^{2} \wedge e^{5} \wedge e^{6}+e^{3} \wedge e^{4} \wedge e^{5} \wedge e^{6}\right) .
\end{aligned}
$$


- Non-vanishing intrinsic torsion classes:

$$
\begin{aligned}
& \mathcal{W}_{1}=-\frac{2 i}{3} \frac{2 a+b}{\sqrt{a^{2} b}} \\
& \mathcal{W}_{2}=-\frac{4 i}{3} \frac{1}{\sqrt{a^{2} b}}\left[a(a-b) e^{12}-2 b(b-a) e^{34}+a(a-b) e^{56}\right] .
\end{aligned}
$$

$G_{2} / S U(3)$

- Metric:

$$
d s^{2}=a\left(e^{1} \otimes e^{1}+e^{2} \otimes e^{2}+e^{3} \otimes e^{3}+e^{4} \otimes e^{4}+e^{5} \otimes e^{5}+e^{6} \otimes e^{6}\right) .
$$

- $S U(3)$-structure:

$$
\begin{aligned}
J & =a e^{1} \wedge e^{2}-a e^{3} \wedge e^{4}-a e^{5} \wedge e^{6}, \\
d J & =-2 \sqrt{3} a\left(e^{1} \wedge e^{3} \wedge e^{5}-e^{1} \wedge e^{4} \wedge e^{6}+e^{2} \wedge e^{3} \wedge e^{6}+e^{2} \wedge e^{4} \wedge e^{5}\right), \\
\Omega & =\sqrt{a^{3}}\left(e^{1}-i e^{2}\right) \wedge\left(e^{3}+i e^{4}\right) \wedge\left(e^{5}+i e^{6}\right), \\
d \Omega & =\frac{1}{\sqrt{3}} 8 i \sqrt{a^{3}}\left(e^{1} \wedge e^{2} \wedge e^{3} \wedge e^{4}+e^{1} \wedge e^{2} \wedge e^{5} \wedge e^{6}-e^{3} \wedge e^{4} \wedge e^{5} \wedge e^{6}\right) .
\end{aligned}
$$

- Non-vanishing intrinsic torsion class:

$$
\mathcal{W}_{1}=-\frac{4 i}{\sqrt{3 a}}
$$

\section{References}

[1] P. Candelas, G. T. Horowitz, A. Strominger and E. Witten, "Vacuum Configurations For Superstrings," Nucl. Phys. B 258, 46 (1985).

[2] A. Strominger, "Superstrings With Torsion," Nucl. Phys. B 274, 253 (1986).

[3] B. de Wit, D. J. Smit and N. D. Hari Dass, "Residual Supersymmetry Of Compactified D = 10 Supergravity," Nucl. Phys. B 283, 165 (1987).

[4] M. Dine, R. Rohm, N. Seiberg and E. Witten, "Gluino Condensation In Superstring Models," Phys. Lett. B 156 (1985) 55.

[5] J. P. Derendinger, L. E. Ibanez and H. P. Nilles, "On The Low-Energy D = 4, N=1 Supergravity Theory Extracted From The D = 10, N=1 Superstring," Phys. Lett. B 155, 65 (1985). 
[6] G. L. Cardoso, G. Curio, G. Dall'Agata, D. Lust, P. Manousselis and G. Zoupanos, "Non-Kähler string backgrounds and their five torsion classes," Nucl. Phys. B 652 (2003) 5 arXiv:hep-th/0211118.

[7] G. Curio and A. Krause, "Four-flux and warped heterotic M-theory compactifications," Nucl. Phys. B 602 (2001) 172 arXiv:hep-th/0012152.

[8] K. Becker, M. Becker, K. Dasgupta and P. S. Green, "Compactifications of heterotic theory on non-Kähler complex manifolds. I," JHEP 0304, 007 (2003) arXiv:hep-th/0301161.

[9] K. Becker, M. Becker, P. S. Green, K. Dasgupta and E. Sharpe, "Compactifications of heterotic strings on non-Kähler complex manifolds. II," Nucl. Phys. B 678, 19 (2004) arXiv:hep-th/0310058.

[10] G. Dall'Agata and N. Prezas, " $\mathrm{N}=1$ geometries for M-theory and type IIA strings with fluxes," Phys. Rev. D 69 (2004) 066004 arXiv:hep-th/0311146.

[11] K. Behrndt and M. Cvetic, "General $\mathrm{N}=1$ supersymmetric fluxes in massive type IIA string theory," Nucl. Phys. B 708, 45 (2005) arXiv:hep-th/0407263.

[12] D. Lust and D. Tsimpis, "Supersymmetric AdS(4) compactifications of IIA supergravity," JHEP 0502 (2005) 027 arXiv:hep-th/0412250.

[13] S. B. Giddings, S. Kachru and J. Polchinski, "Hierarchies from fluxes in string compactifications," Phys. Rev. D 66, 106006 (2002) arXiv:hep-th/0105097.

[14] G. Dall'Agata, "On supersymmetric solutions of type IIB supergravity with general fluxes," Nucl. Phys. B 695, 243 (2004) arXiv:hep-th/0403220.

[15] J. P. Gauntlett, D. Martelli and D. Waldram, "Superstrings with intrinsic torsion," Phys. Rev. D 69, 086002 (2004) arXiv:hep-th/0302158.

[16] A. Gray and L. Hervella, 'The sixteen classes of almost hermitian manifolds and their linear invariants," Ann. Math. Pura, Appl. 123, 35 (1980).

[17] G. L. Cardoso, G. Curio, G. Dall'Agata and D. Lust, "Heterotic string theory on nonKaehler manifolds with H-flux and gaugino condensate," Fortsch. Phys. 52, 483 (2004) arXiv:hep-th/0310021.

[18] A. R. Frey and M. Lippert, "AdS strings with torsion: Non-complex heterotic compactifications," arXiv:hep-th/0507202. 
[19] L. Castellani and D. Lust, "Superstring Compactification On Homogeneous Coset Spaces With Torsion," Nucl. Phys. B 296, 143 (1988).

[20] D. Lust, "Compactification Of Ten-Dimensional Superstring Theories Over Ricci Flat Coset Spaces," Nucl. Phys. B 276, 220 (1986).

[21] T. R. Govindarajan, A. S. Joshipura, S. D. Rindani and U. Sarkar, "Coset Spaces As Alternatives To Calabi-Yau Spaces In The Presence Of Gaugino Condensation," Int. J. Mod. Phys. A 2, 797 (1987).

[22] T. R. Govindarajan, A. S. Joshipura, S. D. Rindani and U. Sarkar, "Supersymmetric Compactification Of The Heterotic String On Coset Spaces," Phys. Rev. Lett. 57, 2489 (1986).

[23] K. Konishi, N. Magnoli and H. Panagopoulos, "Spontaneous Breaking Of Local Supersymmetry By Gravitational Instantons," Nucl. Phys. B 309 (1988) 201.

[24] K. Konishi, N. Magnoli and H. Panagopoulos, "Generation Of Mass Hierarchies And Gravitational Instanton Induced Supersymmetry Breaking," Nucl. Phys. B 323 (1989) 441.

[25] N. Kitazawa, "Gravitino condensation in fivebrane backgrounds," Phys. Rev. D 65, 086004 (2002) arXiv:hep-th/0108232.

[26] M. J. Duff and C. A. Orzalesi, "The Cosmological Constant In Spontaneously Compactified D = 11 Supergravity," Phys. Lett. B 122, 37 (1983).

[27] J. P. Gauntlett and S. Pakis, "The geometry of D = 11 Killing spinors," JHEP 0304 (2003) 039 arXiv:hep-th/0212008.

[28] S. Kachru, R. Kallosh, A. Linde and S. P. Trivedi, "De Sitter vacua in string theory," Phys. Rev. D 68, 046005 (2003) arXiv:hep-th/0301240.

[29] G. Curio and A. Krause, "G-fluxes and non-perturbative stabilisation of heterotic Mtheory," Nucl. Phys. B 643 (2002) 131 arXiv:hep-th/0108220.

[30] C. P. Burgess, R. Kallosh and F. Quevedo, "de Sitter string vacua from supersymmetric D-terms," JHEP 0310, 056 (2003) arXiv:hep-th/0309187.

[31] D. Lust and G. Zoupanos, "Dimensional Reduction Of Ten-Dimensional E8 Gauge Theory Over A Compact Coset Space S/R," Phys. Lett. B 165, 309 (1985). 
[32] P. Manousselis and G. Zoupanos, "Dimensional reduction of ten-dimensional supersymmetric gauge theories in the $\mathrm{N}=1, \mathrm{D}=4$ superfield formalism," JHEP 0411, 025 (2004) arXiv:hep-ph/0406207.

[33] P. Manousselis and G. Zoupanos, "Dimensional reduction over coset spaces and supersymmetry breaking," JHEP 0203, 002 (2002) arXiv:hep-ph/0111125.

[34] P. Manousselis and G. Zoupanos, "Soft supersymmetry breaking due to dimensional reduction over non-symmetric coset spaces," Phys. Lett. B 518, 171 (2001) arXiv:hep-ph/0106033.

[35] P. Manousselis and G. Zoupanos, "Supersymmetry breaking by dimensional reduction over coset spaces," Phys. Lett. B 504, 122 (2001) arXiv:hep-ph/0010141.

[36] E. Bergshoeff, M. de Roo, B. de Wit and P. van Nieuwenhuizen, "Ten-Dimensional Maxwell-Einstein Supergravity, Its Currents, And The Issue Of Its Auxiliary Fields," Nucl. Phys. B 195, 97 (1982).

[37] G. F. Chapline and N. S. Manton, "Unification Of Yang-Mills Theory And Supergravity In Ten-Dimensions," Phys. Lett. B 120 (1983) 105.

[38] E. A. Bergshoeff and M. de Roo, "The Quartic Effective Action Of The Heterotic String And Supersymmetry," Nucl. Phys. B 328, 439 (1989).

[39] P. Manousselis, N. Prezas and G. Zoupanos, work in progress.

[40] V. A. Novikov, M. A. Shifman, A. I. Vainshtein and V. I. Zakharov, "Instanton Effects In Supersymmetric Theories," Nucl. Phys. B 229 (1983) 407.

[41] V. A. Novikov, M. A. Shifman, A. I. Vainshtein, M. B. Voloshin and V. I. Zakharov, "Supersymmetry Transformations Of Instantons," Nucl. Phys. B 229 (1983) 394.

[42] P. G. Camara, A. Font and L. E. Ibanez, "Fluxes, moduli fixing and MSSM-like vacua in a simple IIA orientifold," arXiv:hep-th/0506066.

[43] G. Dall'Agata and N. Prezas, "Scherk-Schwarz reduction of M-theory on $\mathrm{G}_{2}$-manifolds with fluxes," JHEP 0510 (2005) 103 arXiv:hep-th/0509052.

[44] M. B. Green and J. H. Schwarz, "Anomaly Cancellation In Supersymmetric D=10 Gauge Theory And Superstring Theory," Phys. Lett. B 149, 117 (1984).

[45] C. M. Hull, "Compactifications Of The Heterotic Superstring," Phys. Lett. B 178 (1986) 357. 
[46] C. M. Hull, "Anomalies, Ambiguities And Superstrings," Phys. Lett. B 167, 51 (1986).

[47] P. Forgacs and N. S. Manton, "Space-Time Symmetries In Gauge Theories," Commun. Math. Phys. 72, 15 (1980).

[48] Y. A. Kubyshin, I. P. Volobuev, J. M. Mourao and G. Rudolph, "Dimensional Reduction Of Gauge Theories, Spontaneous Compactification And Model Building".

[49] D. Kapetanakis and G. Zoupanos, "Coset space dimensional reduction of gauge theories," Phys. Rept. 219, 1 (1992).

[50] F. A. Bais, K. J. Barnes, P. Forgacs and G. Zoupanos, "Dimensional Reduction Of Gauge Theories Yielding Unified Models Spontaneously Broken To SU(3) X U(1)," Nucl. Phys. B 263, 557 (1986).

[51] D. Kapetanakis and G. Zoupanos, "Constructing Unified Models Based On E(8) In Ten-Dimensions," Z. Phys. C 56, 91 (1992).

[52] A. Micu, "Heterotic compactifications and nearly-Kaehler manifolds," Phys. Rev. D 70 (2004) 126002 arXiv:hep-th/0409008.

[53] U. Gran, "GAMMA: A Mathematica package for performing Gamma-matrix algebra and Fierz arXiv:hep-th/0105086.

[54] T. House and E. Palti, "Effective action of (massive) IIA on manifolds with SU(3) structure," arXiv:hep-th/0505177.

[55] F. Mueller-Hoissen and R. Stuckl, "Coset Spaces And Ten-Dimensional Unified Theories," Class. Quant. Grav. 5, 27 (1988).

[56] J. Gutowski, S. Ivanov and G. Papadopoulos, "Deformations of generalized calibrations and compact non-Kähler manifolds with vanishing first Chern class," arXiv:math.dg/0205012. 